\title{
ESTRATEGIA TURÍSTICA EN EL TRIÁNGULO DEL SOL: ANÁLISIS CON LA TEORÍA BASADA EN LOS RECURSOS Y LA TEORÍA DE LA INSTITUCIONALIDAD
}

\author{
José G. Vargas-Hernández ${ }^{1}$ \\ Universidad de Guadalajara \\ Centro Universitario de Ciencias Económico Administrativas \\ Departamento de Administración \\ josevargas@cucea.udg.mx, jgvh0811@yahoo.com,jvargas2006@gmail.com \\ Gabriela Muratalla-Bautista ${ }^{2}$ \\ Ciencias Económico Administrativas del Instituto Tecnológico del Valle de Morelia \\ gabymuba@outlook.com,gmuratalla@itvallemorelia.edu.mx \\ Ana Yanetly Arias Orozco ${ }^{3}$ \\ Universidad de Guadalajara \\ Centro Universitario de Ciencias Económicas Administrativas \\ yanetly.15.04@gmail.com
}

(Fecha envío: 14/08/16 - Fecha aceptación: 07/12/16)

\section{Resumen}

En el presente trabajo de investigación se abordó el tema de estrategia en el Triángulo del Sol en base al análisis de la teoría basada en los recursos y la teoría de la institucionalidad, por lo que se realizó un estudio descriptivo y explicativo se describen los conceptos relacionados con los elementos antes mencionados y se explica la derrama económica, el tipo y cantidad de turistas. El método que se llevó a cabo fue de buscar información de investigaciones teóricas y empíricas, posteriormente se obtuvieron datos estadísticos referentes a los ingresos que se perciben por la actividad económica por el turismo en los años correspondientes del 2005 al 2015, así como el tipo de personas que visitan a este sitio en el mismo periodo. En los resultados principales se identificó que en el año 2013 se tiene un decremento de más del $50 \%$ considerable en la derrama económica y la cantidad de turistas, concluyendo que esto que la estrategia implementada por los poderes ejecutivos del Estado de Guerrero no ha tenido un impacto positivo en el sector turístico.

\section{Palabras clave}

estrategia, teoría de los recursos, teoría de la institución, declive turístico, Triángulo del Sol

\section{Abstract}

In the present research work the subject of strategy in the Triangle of the Sun was approached based on the analysis of the theory based on the resources and the theory of the institutionality, so a descriptive and explanatory study was carried out describing the related concepts with the aforementioned elements and explains the economic pumping, the type and quantity of tourists. The method used was to search for information from theoretical and empirical research. Later, statistical data were obtained on the income received by the economic activity by tourism in the corresponding years from 2005 to 2015, as well as the type of People who visit this site in the same period. In the main results, it was identified that in the year 2013 there is a significant decrease of over $50 \%$ in the economic outlay and the number of tourists, concluding that the strategy implemented by the executive powers of the State of Guerrero has not had a Positive impact on the tourism sector.

\section{Key Words}

strategy, resources theory, theory of the institution, tourist decline, Triángulo del Sol

\footnotetext{
${ }^{1}$ M.B.A.; Ph. D.

${ }^{2} \mathrm{M}$. en $\mathrm{A}$.

3 Lic.
} 
JEL

H10, H53, D78

\section{INTRODUCCIÓN}

En los últimos años el turismo ha presentado una marcada tendencia de crecimiento nivel internacional, convirtiéndose para muchos países en una de las actividades económicas principales de ellos, debido a los impactos positivos que tienen en el desarrollo y crecimiento de una nación. "El sector económico del turismo se ha convertido en los últimos años en el sector económico de mayor importancia en cuanto a la generación de empleo, de exportación y de estímulo de inversión y crecimiento económico" (Schulte, 2003, p. 8).

Esto ha permitido que el turismo sea estudiado por investigadores recalcando la importancia y los beneficios que puede generar. Para Santana (1997) los impactos generados por la actividad turística pueden englobarse en tres categorías: el impacto económico, estarán en función de factores como la creación de riqueza y de su distribución, de la mano, los costes y beneficios producidos, obra empleada de población local o extranjera. El impacto físico o ambiental es decir las consecuencias que pueda generarse en el medio ambiente dada las actividades turísticas. Y por último el impacto cultural y social en las formas de vida de la población.

La Organización Mundial del Turismo ha exhortado a los todos los países del mundo para que apoyen y promuevan dicho sector. México ha sido uno de los países que ha implementado estos consejos, convirtiéndose en el noveno país que más turistas recibe en el mundo y el lugar 17 por los ingresos obtenidos por esta actividad (DATATUR, 2016). El sector turismo es considerado como un factor prioritario para el desarrollo nacional, debido a su elevada productividad y a la capacidad de generación de empleos (Meixueiro, 2008). La participación turística en el Producto Interno Bruto (PIB) para el año 2014 fue de 8.6 \%, empleando a 39'541,248 y divisas por 16 mil 257.9 millones de dólares (INEGI, 2014).

El actual gobierno federal también ha propuesto el impulso de este sector como uno de principales objetivos del Plan de Desarrollo Nacional 2012-2018, teniendo como propósito la realización de 10 metas en este rubro, así como la implementación de las respectivas estrategias que se utilizarían. Las cuales van desde la promoción de los destinos turísticos, hasta la elaboración de diagnósticos por parte de universidades que identifiquen los principales problemas de los 44 destinos considerados como los más importantes del país.

El triángulo del Sol ha sido uno de los destinos turísticos en donde se han llevado a cabo la implementación de dichas estrategias, para ello se ha tenido la participación de los tres niveles de gobierno, teniendo un presupuesto asignado de 272 mil 240,400 millones de pesos para secretaría de Turismo para la promoción y fomento del turismo del Estado de Guerrero en el 2016.

Por lo mencionado anteriormente se conforma el objetivo de la investigación que es determinar el impacto que tiene la estrategia turística utilizada por el Triángulo del Sol destino turístico del Estado de Guerrero.

\section{ANTECEDENTES DEL PROBLEMA}

El Triángulo del Sol, es la zona turística más importante del estado de Guerrero, esta región está conformada por los municipios de Acapulco de Juárez, Taxco de Alarcón y el binomio de Ixtapa-Zihuatanejo, la ubicación geográfica de cada uno forman lo que pareciera un triángulo; se caracterizan porque la mayoría de los días hay sol, de ahí la nomenclatura, como se puede observar en la figura 1.

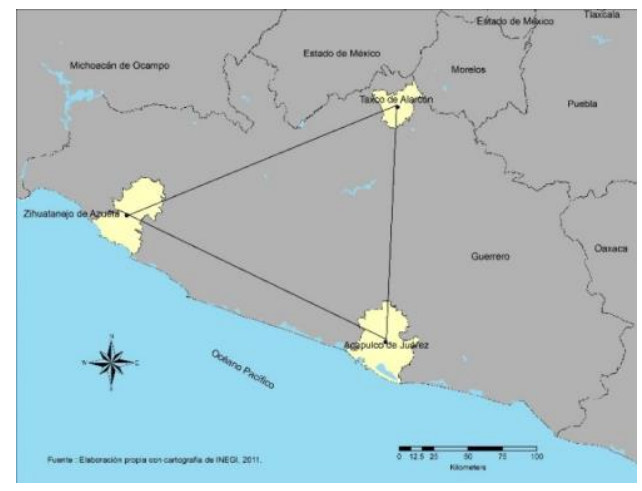

Figura 1: Ubicación geográfica del Triángulo del Sol

Fuente: Elaboración propia con cartografía de INEGI, 2011. 
Taxco ha sido conocido desde los años de virreinato por su excelsa producción de plata, y siendo está su principal actividad económica. En el año del 2002 fue decretado como pueblo mágico, por lo cual el turismo se convirtió en la segunda actividad más importante. En los años 1970-1979 mediante un préstamo realizado con Banco Interamericano de Desarrollo fue creado el destino turístico Ixtapa-Zihuatanejo, el cual tiene un poco más de 40 años de su surgimiento junto con Cancún. Uno de los principales destinos turísticos es Acapulco el más conocido de los tres, desde los años 50 s y 70 s vivió un auge en este sector, y durante los años siguientes fue uno de los lugares preferidos por los turistas a nivel nacional e internacional.

El Triángulo del Sol refleja un declive en el turismo causando a su vez un estancamiento económico en la región. El declive tiene diferentes explicaciones por expertos en el tema, siendo para unos la inseguridad el factor más importante, ya que el estado de Guerrero ha estado sumergido durante los últimos 10 años en violencia consecuencia de la guerra contra el narcotráfico.

Sin embargo, este declive surgió mucho antes de que se profundizara la violencia, pues Acapulco mostraba indicios de esta problemática desde finales de los años $80 \mathrm{~s}$, además de que la afluencia de los otros municipios siempre fue en menor proporción con respecto Acapulco. Por lo que la inseguridad simplemente agudizo e hizo más notoria la situación que estaba viviendo el Triángulo del Sol. El declive turístico ha generado impactos en la derrama económica, en el empleo, en la afluencia de turistas que visitan el lugar, es decir impactos económicos y sociales.

Algunos posibles factores que podrían explicar esta problemática del declive de esta región son surgimientos de otros destinos turísticos, los problemas ambientales que tienen estos lugares, la inestabilidad económica del estado, la atención tardía de las autoridades (Bringas, 1999). Los documentos que hablen en conjunto de estos municipios son muy pocos, la mayoría se centra en Acapulco por ser el más conocido y durante mucho tiempo el número uno tanto nacional como internacionalmente.

Recientemente han surgido teorías que consideran que el turismo tiene un ciclo de vida y hasta cierto punto explican cómo algunos destinos turísticos llegan al declive, una de ellas expuesta por Butler (1980) el cual sostiene que el un destino atraviesa diferentes fases: explotación, implicación, desarrollo, consolidación, estancamiento y declive.

Una pronunciada diferenciación que hay entre el estancamiento y el declive turístico es que el segundo se caracteriza por la reducción significativa de la captación de turistas, mientras que en el estancamiento se percibe un promedio de turistas, es decir como si el flujo de turistas fuera constante.

En la fase del declive es necesario que se tomen medidas para volver a ser atractivo el destino. Es aquí donde también se tienen que retomar la idea de buscar otras nuevas atracciones, renovación o cualquier medio que permita rejuvenecer a este sector. Esta teoría es muy criticada por su simplicidad, pero es la más utilizada debido a ser la más explicativa en cuanto a destinos.

\section{DELIMITACIÓN DEL PROBLEMA}

A pesar de las fuertes inyecciones de dinero por parte del gobierno que se le ha hecho a la estrategia para la promoción de estos destinos y mejoramiento de infraestructura en cada uno de los municipios, los índices socioeconómicos no han sido favorables.

Lo que genera la pregunta de investigación:

¿Es un fracaso la estrategia turística utilizada por el Triángulo del Sol?

Principalmente por la infinita cantidad de recursos que tienen los tres municipios y que para Peng (2012) el aprovechamiento de estos es el desarrollo de una estrategia efectiva. Mientras que las instituciones son "las reglas del juego" North (1990), en donde en este caso quienes han llevado a cabo la implementación y ejecución de la estrategia.

\section{JUSTIFICACIÓN}

La importancia de este sector de estos municipios es de crucial importancia, en el cual los tres niveles de poder están centrando su atención, al no obtener resultados esperados podría perjudicar a la población, entonces analizar este tema permitirá evitar este tipo de cosas o por lo menos proponer algo para mejorar la situación.

La relevancia de este trabajo permite tener una visión desde otra perspectiva, aplicando teorías que son fundamentales a la hora de llevar a cabo una estrategia, y más de este tipo de magnitud, donde no sólo está en juego el bienestar de la población de la región sino también de la economía nacional. Los trabajos de 
investigación existentes sobre estos municipios para saber la situación en la que se encuentran son principalmente diagnósticos, además del aborde individualmente no en su conjunto.

\section{MARCO TEÓRICO}

En este apartado primeramente se conceptualiza la estrategia turística, para luego precisar las teorías que aplican al objeto de estudio de la investigación que en este caso con las estrategias turísticas en el Triángulo del Sol. Posteriormente se realizó la revisión de la literatura teórica e empírica sobre la teoría basada en los recursos. Las investigaciones que se ha efectuado y se relacionan con la teoría de la institucionalidad.

\subsection{Estrategia turística}

Con el fin de facilitar la comprensión del presente texto, a continuación, se proporcionan algunos conceptos de estrategias turísticas.

"La estrategia se define como el alineamiento o dirección que se dan a los recursos internos de una organización para modificar, liderar, adaptarse y en el peor de los casos sobrevivir a las condiciones del medio ambiente" (Vargas-Hernández y Bojórquez-Gutiérrez, 2014, p. 61).

Una estrategia turística para que sea eficiente por tanto tienen que tener esa capacidad de adaptación para poder sobrevivir a los constantes cambios, primordialmente al mercado tan dinámico y competitivo que es, y en donde el turismo también tiende a ser parte de ellos.

Algunas de las estrategias que el gobierno ha intentado implementar para reactivar en el turismo en el país, y que el gobierno del Estado de Guerrero lleva de igual manera son:

a. Fortalecer las capacidades institucionales del sector turismo

b. Impulsar la coordinación intergubernamental en materia turística

c. Fortalecer los mecanismos de concertación con la academia, el sector privado y el sector social, en beneficio del turismo

d. Generar información, investigación y conocimiento sobre los destinos y líneas de producto

e. Impulsar la innovación, diversificación y consolidación de la oferta turística por región y destino

f. Promover altos estándares de calidad en los servicios turísticos

g. Impulsar la profesionalización de los prestadores de servicios

h. Impulsar una política de seguridad integral para proporcionar una

i. Facilitar el financiamiento y la inversión público - privada

Se realizó un análisis de las estrategias del Programa Nacional de Desarrollo actual con los otros y se encontró que no se han efectuado grandes cambios con respecto a las estrategias, estás siguen siendo iguales nada más con diferentes palabras, los cambios son mínimos la única diferencia de mayor importancia son los acuerdos de profesionalización que se han hecho para ver la situación actual en la que se encuentran cada uno de los destinos. Así como en los últimos en los 12 años de los informes de gobierno del estado de Guerrero y sucede la misma situación.

Es importante mencionar que durante los últimos años la inversión pública para el mejoramiento de la infraestructura de estos municipios, así como la promoción y difusión en medios de los tres destinos ha sido excesivo. Durante el 2013 la Secretaría de Turismo destino 25 millones de pesos para la promoción del destino turístico y durante los años de 2012- 2014 se invirtió 735 millones de pesos en el estado de obras de infraestructura para los tres municipios y en el 2016 se destinó 11 millones (El Universal, 2016).

\subsection{Revisión teórica y empírica de la Teoría de los recursos}

La teoría de los recursos pone énfasis en el aprovechamiento de los recursos internos y su gestión, así como a las capacidades dinámicas que se puedan tener (Penrose, 1959). Los recursos son definidos como los bienes o activos tangibles e intangibles que usan una forma para elegir e implementar sus estrategias. Los tangibles son aquellos que pueden ver y cuantificar fácilmente mientras que los intangibles son aquellos que son difíciles de ver cuantificar (Peng, 2012).

Los recursos tangibles con los que cuenta los municipios Acapulco de Juárez e Ixtapa-Zihuatanejo se caracterizan por hermosas playas turísticas, mientras que Taxco de Alarcón por la belleza arquitectónica y su producción de plata. Y los tres lugares cuentan con museos, teatros, plazas, bares, antros y demás lugares de esparcimiento social. En el caso de los recursos tangibles financieros podemos mencionar los recursos destinados por parte del gobierno federal y estatal para la toda la implicación que requiere la promoción de estos lugares. 
En cuanto a recursos intangibles es la calidez de personas que la habitan y atienden, en cuanto a su innovación sería el mejoramiento de infraestructura, desafortunadamente su reputación de seguridad no es muy buena, esto le ha afectado seriamente, el capital humano de la población de cada municipio.

Cancún se posiciono como el lugar preferido para los vacacionistas, los recursos con los que cuenta a diferencia de Ixtapa-Zihuatanejo son más diversificados, además de contar con playas y lugares de diversión, cuenta con algo que poco tienen el triángulo del sol, las zonas arqueológicas tan milenarias, además de una cultura tan importante como lo fue la maya.

Las estrategias genéricas en estos municipios han sido aplicables dado que, aunque no ha sido el líder en precios ha intentado utilizado o reducido los costos de habitación y hasta han firmado acuerdos con aerolíneas para que ofrezcan vuelos más baratos, de hecho, los precios están por debajo de destinos similares, sin embargo, no ha funcionado del todo esta estrategia.

La diferenciación que han intentado hacer es ofrecer los destinos de Taxco e Ixtapa- Zihuatanejo como uno de los destinos ideales para descansar y con unas bellezas naturales, pero con los sucesos ocurridos de violencia esto ha tenido poco impacto. En cuanto al enfoque, debido a la reducación de turistas extranjeros se ha promovido o enfocado al mercado nacional, sobretodo ofreciéndolo principalmente con los estados vecinos del estado de Guerrero, convirtiéndose en un lugar más visitado por los nacionales.

En la investigación desarrollada por Cardona (2011, p. 141), sobre la estrategia basada en los recursos y capacidades. criterios de evaluación y el proceso de desarrollo concluye que las empresas u organizaciones "pueden obtener ventajas competitivas y beneficios en forma sostenida, siempre y cuando se disponga de recursos únicos, de acuerdo a los criterios usados para valorarlos, a su relación con los factores claves de éxito".

En este estudio ya no se aplicó el análisis FODA (Fortalezas, Oportunidades, Debilidades y Amenazas) debido a que los estudios encontrados ya lo han abordado y lo que aquí se pretenden es elaborar algo nuevo, además es el análisis se aplica en todos sus informes de gobiernos del estado de Guerrero.

\subsection{Revisión teórica y empírica de la Teoría de la Institucionalidad}

Para Schutter (1981) la encargada de la conducta social aceptada socialmente será la institución, que a su vez crea las tanto de manera interna como externa las políticas. En esta teoría también el estado del Estado juega un papel importante pues tratan de reducir la incertidumbre, de diferentes maneras, políticamente, económicamente, contratos, transacción.

En una sociedad los factores tanto formales como informales del contexto institucional provocan cambios económicos, dando con ello el crecimiento y desarrollo del estado. Además, la teoría institucional ha incorporado ganancias en el análisis de costos de transacción, en las organizaciones las reglas y aspectos que impactan en la eficiencia, estructura de gestión, así como las normas informales de los diferentes grupos (Macagnan, 2013).

Haciendo el análisis con el triángulo del sol se puede observar que el Estado no supo reducir esa incertidumbre, pues políticamente en los últimos ha estado lleno de tensión, en primer lugar, por la violencia del narcotráfico y segundo los hechos ocurridos en Ayotzinapan, los que aparte de demostrar la ineficiencia de las instituciones en los tres niveles terminaron de dañar la reputación del Estado, considerándolo el más violento del país.

Económicamente los costos de transacción para las empresas que han querido invertir no se han reducido, lo cual una negociación con ese estado sale muy costosa, lo que lleva a mejor no querer invertir. Es preciso mencionar que se calcula que se han cerrado un aproximadamente de 1,500 empresas por la poca rentabilidad en la región, afectando a la economía.

Las instituciones han participado en conjunto desde los tres niveles de gobiernos, así sin embargo al parecer no ha habido esa vinculación suficiente para poderse coordinar, lo cual también afecta a la ejecución de la estrategia y por ende la dependencia que pueda tener el éxito.

En la línea argumentativa de la teoría institucional esta el desempeño que tienen los diferentes roles que se juegan en las instituciones $u$ organizaciones enfatizan en un aspecto central que guían su operación por medio de reglas, valores y actitudes que retoman del medio ambiente donde se encuentren ubicadas, pretendiendo que esta manifestación de alineamiento les permita conservar el poder y los intereses que tienen (Alvarado 2006).

Por otro lado, un rol estratégico como lo menciona Vargas-Hernández y Bojórquez-Gutiérrez (2014) es la ética, cosa que desafortunadamente las instituciones no han sabido ofrecer, pues han existido muchas irregularidades en cuanto a la declaración de los gastos efectuados en los eventos que realizan para promover los puertos, así como el concurrido nepotismo. Tan poca es la ética que el encargado actual de la Secretaria de Turismo del estado de Guerrero es una persona sin formación profesional, lo cual lo deja fuera de tener la experiencia, pero sobre todo el capital humano que requiere tener alguien en un cargo tan importante como el que él preside. Lejos de contribuir la ética aquí la corrupción, es que de la segunda hay mucha y la primera no existe. 


\section{MÉTODO}

En esta investigación es de tipo descriptivo y explicativo, ya que se describen los conceptos relacionados con las estrategias turísticas, la teoría basaba en los recursos y la teoría de la institucionalidad en el Triángulo del Sol centro turístico que se encuentra ubicado en el Estado de Guerrero, ya que el principal objetivo es determinar el impacto que tiene la estrategia turística utilizada por dicho centro, así como el relacionar las diferentes teorías organizacionales que se aplican para generar el cambio y tener un impacto positivo en el Triángulo del Sol, como lo mencionan Münch y Ángeles (2012, p. 57) "el marco teórico o marco conceptual es la exposición y análisis de la teoría o grupo de teorías que sirven como fundamento para explicar los antecedentes e interpretar los resultados de la investigación".

Para poder efectuar el presente trabajo se llevó a cabo el procedimiento de buscar información de investigaciones teóricas y empíricas, posteriormente se obtuvieron datos estadísticos referentes a los ingresos que se perciben por la actividad económica por el turismo en los años correspondientes del 2005 al 2015, así como el tipo de personas que visitan a este sitio en el mismo periodo.

\section{RESULTADOS}

La derrama economica en el Triángulo del Sol en los últimos años ha presentado un comportamiento irregular, pero sobretodo el norotio decenso que ha tenido en los dos ultimos periodos, son muy pocas las recuperaciones que ha tenido, de hecho si se observa a detalle la figura 2 se puede observar que en el año 2005 se tiene una derrama de 42, 474 miles de millones de dolares, en el año 2006 hay un incremento y se obtine 46,829 miles de millones y por último en el 2007 tambien se tiene un incremento de 47,588 millones, pero a partir del 2007 comienza un declive pronunciandose más en 2015.

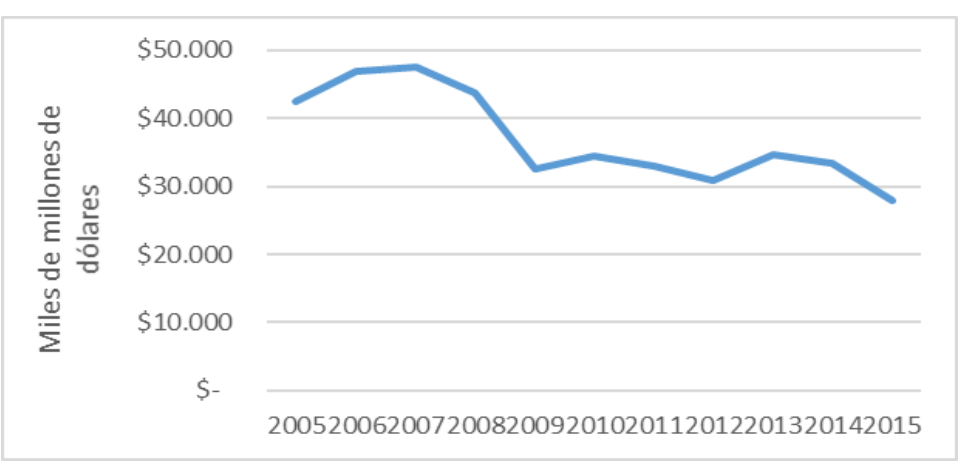

Figura 2: Derrama económica del Triángulo del Sol, 2000-2015

Fuente: Elaboración propias con informes de gobierno del Estado de Guerrero, 2005-2015

En la figura 3 se muestran los turistas que visitaron el Tringulo del Sol, la cual refleja diferentes variaciones por ejemplo durante los periodos comprendidos del 2005 al 2007 tiene una tendencia positiva, pero a partir del 2008 se convierte en negativa teniendo un fuerte descenso en el año 2012 al 2013 del más del 50\%. Se estabiliza en el año 2014 logrando mantenerse en esa misma tendencia, ya que los turistas que lo visitan gastan cantidades considerables, lo cual impacta en la derrama económica.

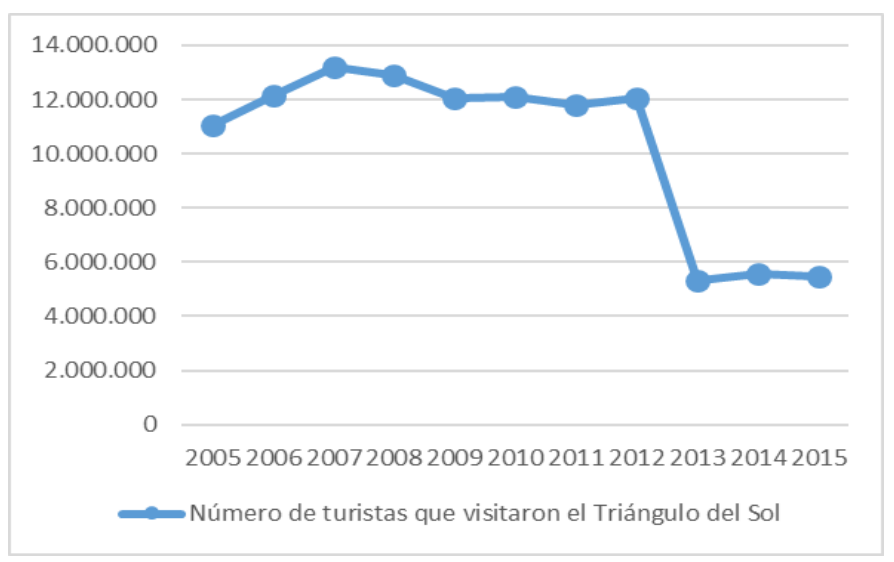

Figura 3: Turisistas que visitaron el Tringulo del Sol

Fuente: Elaboración propias con informes de gobierno del Estado de Guerrero, 2005-2015 
El gobierno ha realizado diferentes eventos que impactan de forma directa a la derrama económica, ya que en época de vacaciones la ocupación hotelera no ha sido al 100\%, hasta el 2016 se ha comenzado a recuperar, esto lo manifiestan los turistas que han visitado es esos periodos y han tenido la oportunidad de disfrutar de esos eventos. Con principios económicos se sabe que, al no haber un alto número de turistas, no se generan fuentes de trabajo y por ende el consumo también disminuye.

Mientras que en la figura 4 muestra el tipo de visitantes que recibió el Triángulo del Sol, se puede observar claramente la brecha tan grande que hay entre los turistas nacionales y los extranjeros que lo visitan, presentando también una caída muy fuerte en el año 2013 principalmente en el turismo nacional.

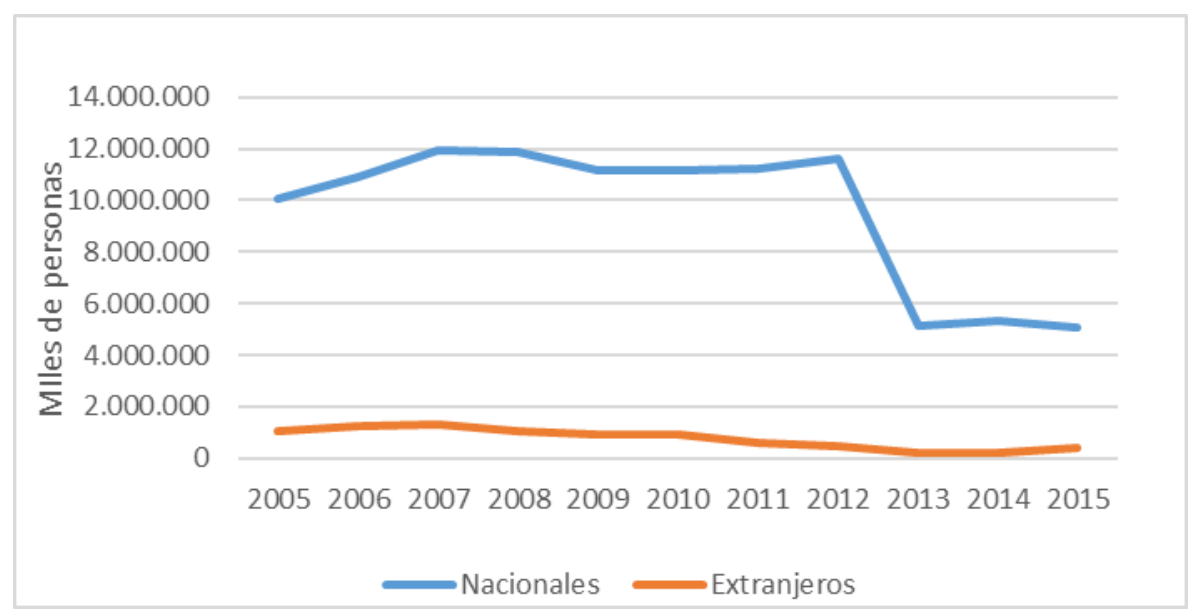

Figura 4: Tipo de visitantes que recibió el Triángulo del Sol, 2005-2015

Fuente: Elaboración propias con informes de gobierno del Estado de Guerrero, 2005-2015

Referente al trabajo cabe mencionar que muchas personas se quedaron sin empleo por la poca demanda turística, y en algunos hoteles se aplicó la estrategia de reducir los sueldos para que los trabajadores siguieran teniendo una fuente de ingresos, aunque menor pero constante. Esto se logró debido a que la gran mayoría de los hoteles ubicados en Acapulco e Ixtapa son cadenas de hoteles distribuidos por toda la república y los ingresos de otros destinos podían permitirles tomar estas medidas.

En cuanto a índices socioeconómicos se presentan algunos datos en la tabla 1 que reflejan la situación, es preciso decir que estos datos están basados en 2010 pues es la información estadística oficial más reciente:

\begin{tabular}{|l|c|c|c|}
\hline \multicolumn{1}{|c|}{ Indicadores } & Acapulco & Ixtapa- Zihuatanejo & Taxco \\
\hline Pobreza & $51.6 \%$ & $53.6 \%$ & $59.2 \%$ \\
\hline Rezago educativo & $19.2 \%$ & $23.2 \%$ & $25 \%$ \\
\hline Servicios de salud & $39.3 \%$ & $31.3 \%$ & $36.4 \%$ \\
\hline $\begin{array}{l}\text { Población ocupada con } \\
\text { ingreso de hasta 2 } \\
\text { salarios mínimos }\end{array}$ & $42.68 \%$ & $34.85 \%$ & $54.30 \%$ \\
\hline
\end{tabular}

Tabla 1: Indicadores socioeconómicos del Triángulo del Sol, 2010

Fuente: Elaboración propia con datos de CONAPO, 2010.

Como se puede observar los resultados en estos aspectos son poco alentadores, lo cual indica la situación económica en que se encuentran estos tres municipios, producto del declive turístico y su repercusión en la economía del estado. Para Matthews (1986) las instituciones afectan a las personas en sus vidas económicas, pues son ellas las que tienen tanto derechos como obligaciones, que al final implican en ellas.

En este caso se puede ver que han tenido consecuencias económicas y sociales. Esto es que la estrategia del estado ha tenido muy poco éxito, ya que en la actualidad las condiciones se encuentran, ya que no se ha logrado el incremento deseado, dando con ello un área de oportunidad de analizar otros sectores que podrían ser más funcionales que este. Como dice Barney (2008), a veces es necesario buscar cadenas de valor, que fortalezcan las actividades que ya se tienen y generar otras nuevas en el estado de Guerrero.

\section{CONCLUSIONES}

Analizando la situación y los resultados se puede concluir que la estrategia turística utilizada por el gobierno del estado de Guerrero realmente no ha tenido el impacto que el gobierno esperaba, logrando con ello el objetivo planteado en la presente investigación que fue el determinar el impacto que tiene la estrategia turística utilizada 
por el Triángulo del Sol destino turístico del Estado de Guerrero, ya que no ha dado los resultados pretendidos, además de que la población es seriamente afectada al no ser exitosa. La inversión que están haciendo no se ve equilibrada en los resultados económicos y mucho menos con los sociales.

Aun cuando se han elaborado diagnósticos para atender esta problemática, la verdad es que no se han efectuado con la profundidad que se requiere en esa región para encontrar cuál es la verdadera razón que origina esta situación. Es cierto que la inseguridad tiene un peso muy importante para contribuir al fracaso de las estrategias implementadas, más sin embargo hay que reconocer que el declive se presentó mucho antes y que las autoridades encargadas tomaron las decisiones en la fase más crítica del problema. En ocasiones las problemáticas sociales son como las enfermedades de salud, debe de recibir un diagnóstico y tratamiento en tiempo y forma para evitar llegar a una fase terminal.

Las sugerencias que se hacen son:

a. Hacer un análisis completo de los factores posibles de esta situación

b. Buscar nuevos sectores o actividades económicas en donde cada uno de estos municipios podría estar mejor

c. Participar con varios expertos no sólo en economía, turismo, sino en otras ciencias sociales, sociología, geógrafos, etc. para permitirles un escenario más amplio.

Poder llevar a cabo el turismo como una actividad económica es necesario tener en cuenta que debe permitir el desarrollo (OMT, 2016).

\section{REFERENCIAS BIBLIOGRÁFICAS}

Alvarado, S. (2006). Metamorfosis de la concepción del cambio organizacional en el nuevo institucionalismo. Contaduría y Administración. No. 219, mayo-agosto, pp. 11-40. Disponible en: http://www.redalyc.org/articulo.oa?id=39521901

Barney, J. (2008). Chapter 3: Evaluating a firm's internal capabilities, En. Barney, J. (ed.), Strategic Management and competitive advanges (pp. 72-144). New Jersey. Pearson

Bringas, N. (1999). Políticas de desarrollo turístico en dos zonas costeras del Pacífico mexicano. Región y Sociedad vol. XI, núm. 17, enero-junio (pp. 1-50).

Butler, R. (1980). The concept of a tourist area cycle of evolution: implications for management of resources. The Canadian Geographer/Le Géographe canadien, 24(1), pp. 5-12

Cardona, R. (2011). Estrategia basada en los recursos y capacidades. Criterios de evaluación y el proceso de desarrollo. Revista electrónica Forum. Número 4. Pp. 113-147.

CONAPO (2010), Índices de Recuperado de: http://www.conapo.gob.mx/en/CONAPO/Indices_de_Marginacion

DATATUR (2016), Estadísticas, Recuperado de: http://www.datatur.sectur.gob.mx/SitePages/Inicio.aspx

El Universal (2016), Astudillo anuncia inversión de más de un millón para Guerrero. Recuperado de: http://www.eluniversal.com.mx/articulo/estados/2016/01/2/astudillo-anuncia-inversion-de-mas-de-11-milmdp-para-guerrero

INEGI, (2010). Censo de Población y Vivienda 2010. Recuperado de: http://www.inegi.org.mx/est/contenidos/proyectos/ccpv/default.aspx

INEGI, (2011) Cartografía Urbana. Recuperado de: http://www.inegi.org.mx/geo/contenidos/urbana/

INEGI, (2014) PIB $y$ Cuentas Nacionales. Recuperado de: http://www.inegi.org.mx/est/contenidos/proyectos/cn/tur/

Macagnan, C. (2013). Teoría institucional: escrito teórico sobre los protagonistas de la escuela institucionalista de economía. Revista de Administração e Contabilidade da Unisinos. 10(2), pp. 130-141, doi: 10.4013/base.2013.102.03

Matthews, R. C. (1986). The economics of institutions and the sources of growth. The Economic Journal, 96(384), pp. $903-918$

Meixueiro, G. (2008), Impacto de la actividad turística en el desarrollo local, Centro de Estudios Sociales y de Opinión Pública, número 48 (pp. 1-36)

Münch, L. y Ángeles, E. (2012). Métodos y técnicas de investigación. 4ta. Edición. Reimpresión. México: Trillas.

North, D. (1990). Institutions, institutional change and economic performance. Cambridge University Press

OMT (2016). ¿Por qué el turismo? Recuperado de: http://www2.unwto.org/es/content/por-que-el-turismo 
Peng, M. (2012). Global Strategy. Cincinnatu: Thomson South-Western

Penrose, E. (1959). The Theory of the Growth of the Firm. New York: John Wiley

Santana, A. (1997) Antropología del turismo, ¿nuevas hordas, viejas culturas?, Ariel, Barcelona

Schulte. S. (2003). Guía conceptual y metodología para el desarrollo y la planificación del sector turismo. Instituto Latinoamericano y del Caribe de Planificación Económica y Social-ILPES

Schutter, A. (1981). The economic theory of institutions. Cambridge, Cambridge University Press.

Secretaría de Gobierno del Estado de Guerrero (2016), Informes de gobierno 2005-2015. Solicitados a Transparencia: http://guerrero.gob.mx/

Vargas-Hernández, G. \& Bojórquez-Gutiérrez, B. (2014). Gestión estratégica de organizaciones. Ciudad Autónoma de Buenos Aires: Elaleph 